\title{
Persistent currents, flux quantization and magnetomotive forces in normal metals and superconductors
}

\author{
(Review Article) \\ Igor O. Kulik \\ Department of Physics and Astronomy, State University of New York at Stony Brook SUNY \\ Stony Brook, NY 11794-3800, USA \\ E-mail: i.o.kulik@gmail.com
}

Received May 12, 2010

\begin{abstract}
The notion of persistent current comes back to orbital currents in normal metals, semiconductors and even insulators displaying diamagnetic behavior in weak magnetic fields, but came to focus at the discovery of current persistence and magnetic flux quantization at large fields in atomically big but macroscopically small (mesoscopic) objects. The phenomenon bears much similarity with supercurrents in superconductive metals. We will review progress in developing of our understanding of the physical and technological aspects of this phenomenon. The exact solution for currents, magnetic moments and magnetomotive forces (torques) in crossed magnetic fields are presented. Time-dependent phenomena in crossed magnetic and electric fields, and in possibility of spontaneous persistent currents and of work extraction from static and dynamic quantum states are discussed.
\end{abstract}

PACS: 73.23.-b Electronic transport in mesoscopic systems;

03.65.Ta Foundations of quantum mechanics; measurement theory;

71.10.pm Fermions in reduced dimensions (anyons, composite fermions, Luttinger liquid, etc.).

Keywords: Aharonov-Bohm effect, Berry phase, qubit, Coulomb blocade, Luttinger liquid.

\section{Contents}

1. Orbital and spin magnetism in solids: a historical perspective 1057

2. Persistent currents in normal metals, superconductors and dielectrics: a short survey ....................... 1059

3. One-dimensional normal metal ballistic ring in crossed magnetic fields ........................................ 1060

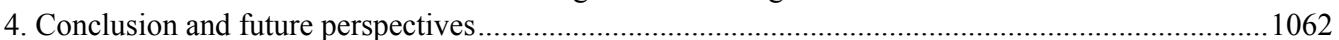

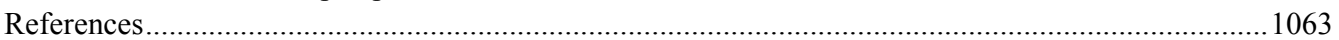

\section{Orbital and spin magnetism in solids:} a historical perspective

Magnetism of solids - metals, insulators and semiconductors - is a pure quantum mechanical property [1]. Orbital magnetism means the existence of nonzero electron current in a state of thermodynamic equilibrium driven by the appliance of external magnetic field. Such nondissipative, non-decaying currents can flow in the superconductive metals at low temperature in presence of static magnetic field [2]. The current may also exist in the nonconducting solids with electrons confined to atoms, molecules or atomic clusters at the lattice sites. Magnetic moment at the site is proportional to the applied magnetic field. The magnetic moment of current $I$ equals (in CGS units)

$$
M=\frac{1}{c} I S,
$$

where $S=\pi R^{2}$ is the effective surface of electron localization perpendicular to the field.

Quantum mechanical computation results in an expression for $M$

$$
M=\frac{Z e^{2}}{4 m_{0} c^{2}} B<\mathbf{r}_{\perp}^{2}>,
$$

where $m_{0}$ if the free electron mass. Magnetic field in a circular loop of radius $R$ equals to $A / R$, where $A$ is the vector potential at the loop whereas an expression in brackets of Eq. (2) is a square of the effective radius, $R$, of the (effective) loop. $\mathrm{Z}$ is the number of electrons in the loop. Assuming dielectric medium of $N$ loops per $\mathrm{cm}^{3}$, 
with the loop size and distance between the loops of order of the Bohr radius, $a_{0}=\hbar^{2} /\left(m_{0} \mathrm{e}^{2}\right)$, we receive magnetic susceptibility of the medium

$$
\chi=\frac{N M}{B}
$$

of order

$$
\chi_{\text {diel }} \sim\left(\frac{e^{2}}{2 \hbar c}\right)^{2} \sim 10^{-5}
$$

at temperature $T=0$. Similar estimate of diamagnetic susceptibility, in case of the bulk normal metal, is

$$
\chi_{\text {metal }} \sim \frac{m_{0}}{m}\left(\frac{e^{2}}{\hbar c}\right)^{2} \sim 10^{-5}
$$

assuming the ratio of the free electron mass to the effective mass of order $10^{-1}$. The expression for magnetic susceptibility of bulk metal

$$
\chi=\frac{1}{3} \mu_{B}^{2} N\left(\mu_{F}\right)=\frac{e^{2} k_{F}}{4 \pi^{2} m c^{2}},
$$

was received by Landau [3] and confirmed by Teller [4] and enters standard courses on quantum mechanics and condensed matter physics [5-8]. Here $k_{F}=2 \pi / a$ is the Fermi wave number, $\mu_{B}=e \hbar / 2 m_{0} c$ is the Bohr magneton, and $N(\varepsilon)$ is the density of electron states at the energy $\varepsilon$ (the estimate of Eq. (5) assumes $a \sim a_{0}$ ).

Approximation made in the derivation of the Eq. (6) rested on the assumption that, in the bulk metal of size $L$ much larger than the Larmor radius $r_{L}=m c v_{F} / e B$, the contribution to the magnetic moment from the edge diamagnetic currents (Fig. 1) is negligible in comparison with the contribution from bulk circular currents. However, as is known from the Van Leeuwen theorem [9], in classical theory both contributions, being of opposite direction of rotation, cancel each other. Quantum calculation in simple geometrical forms ( $2 d$ disks, squares, stripes) showed larger amplitude oscillations [10-14] of magnetic moment compared to the Landau moment, in function of distance to the edge of a sample, in weak magnetic field. In clean metals and strong magnetic fields, thermodynamic and kinetic oscillating phenomena - de Haas-van Alphen effect and Shubnikov-de Haas effect, respectively [6-8], have been discovered and studied revealing a large amount of information on the property of dynamics of mobile electrons considered as elementary excitations (quasiparticles) in metals.

Theoretical prediction by Aharonov and Bohm [15] of an effect, called by their names, of the nonlocal interaction between charged particles and the electromagnetic field such that in certain topological geometries in which magnetic field equals to zero in all space occupied by electrons, but the vector potential is not, the effect of interaction

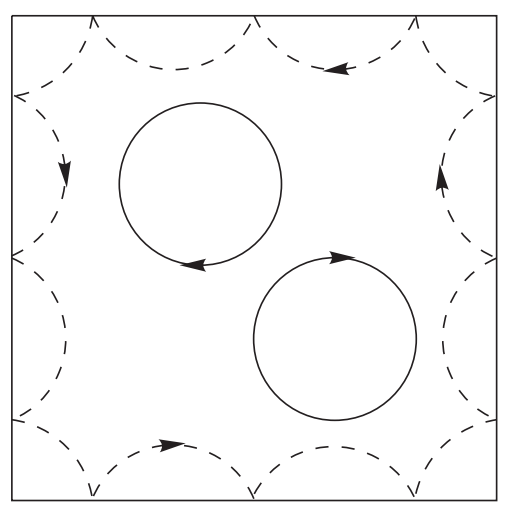

Fig. 1. Electron orbits in a magnetic field for bulk (solid line) and edge state (dashed line) electrons.

shows up and produces the physically observable effects. This stimulated investigations by several authors [16-23], of possible quantum effects in normal metals - the effects similar to the Josephson effect in superconductors. This ended by a prediction in 1970 by the author, of a nondecaying (later called «persistent») currents and flux quantization in a hollow thin-walled normal metallic cylinder and ring [24] threaded by a bunch of flux-lines of magnetic field confined within the inner cylinder (a magnetic coil) of a radius smaller than the radius of outer cylinder (Fig. 2) in which no electric and magnetic field is present. The magnitude of persistent current in normal metal cylinder was estimated as [24]

$$
I \cong \frac{e v_{F}}{L_{1}} \frac{k_{F} L_{2}}{\sqrt{k_{F} L_{1}}} \mathrm{e}^{-T / \varepsilon_{0}} \sin \left(k_{F} L_{1}-\frac{\pi}{4}\right) \sin \left(2 \pi \frac{\phi}{\phi_{0}}\right)
$$

where $\varepsilon_{0}=\hbar v_{F} / L_{1} ; L_{1}$ is the circumference and $L_{2}$ is the length of the cylinder. The current oscillates between diamagnetic and paramagnetic in sign at non-integer value of

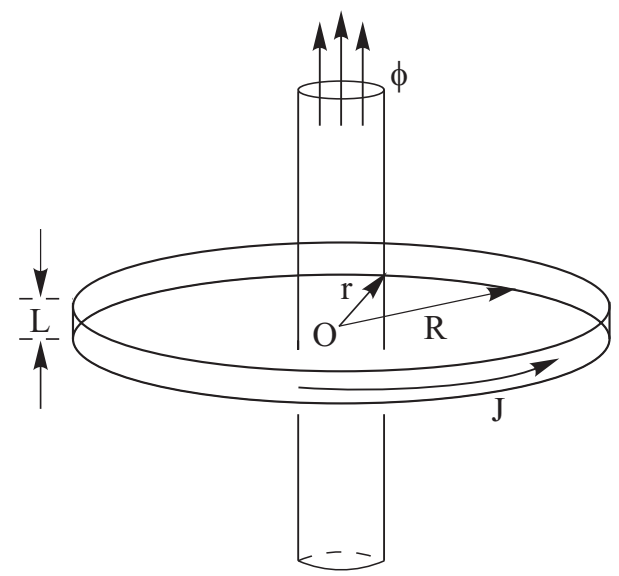

Fig. 2. Schematic of Aharonov-Bohm effect observation in a hollow normal-metal cylinder of length $L$ and radius $R$ threaded by a bunch of lines of force of magnetic field with total magnetic flux $\phi$ confined within an infinitely long cylinder of radius $r<R$ such that the magnetic field outside the cylinder equals to zero but the current in the ring is not. The current varies with the flux periodically with a period $h c / e$. 
$k_{F} L_{1}$ in units of $2 \pi$ in case of fixed chemical potential rather than the fixed particle number. At temperature $T>\varepsilon_{0}$, only the lower flux-oscillating harmonic remains.

At $L_{2}=L_{1}$, Eq. (7) applies to a ring. In that case, the Aharonov-Bohm current estimates as

$$
I_{A B} \sim \frac{e v_{F}}{R} \sin \left(2 \pi \frac{\phi}{\phi_{0}}\right) .
$$

Minimal size of the ring is of order of the Bohr radius $a_{0}$, and in weak field its magnetic moment equals

$$
\mu_{A B}=\mu_{B} \frac{\phi}{\phi_{0}}
$$

will correspond to magnetic susceptibility of $3 d$ insulating medium filled with isolated tightly packed rings

$$
\chi \sim\left(\frac{\mathrm{e}^{2}}{\hbar c}\right)^{2} \sim 10^{-5}
$$

in accordance with an estimate of Eq. (4).

The effect of Aharonov-Bohm persistent current in macroscopic loop was first regarded as doubtful, and at least hardly ready for the experimental realization at the time. Advances in nanotechnology in the next decade revived interest in subtle quantum mechanical phenomena and appliances.

The persistent current was next time considered in 1983 by Buttiker, Imry and Landauer [25] in the normal metallic ring, thus supporting the conclusion of previous papers and effectively stimulating advance in physics of mesoscopic systems and in technological progress of microelectronics.

\section{Persistent currents in normal metals, superconductors and dielectrics: a short survey}

In the time interval between 1970 and 1983, the number of papers discussed possibility of experimental observation of persistent current through its oscillation with the magnetic flux (the flux quantization phenomenon), and extension to different geometric configurations.

In 1981, Altshuler, Aronov and Spivak [26] discovered new oscillating effect in the dirty normal metal with the period $h c / 2 e$ in which a pair of time-reversing electron trajectories at a scattering event interfere and show themselves as $2 e$-charged pair. The effect of $h c / 2 e$ periodic oscillation in the nonsuperconducting melal is a kinetic phenomenon [27] whereas the $h c / e$ periodicity is a thermodynamic Aharonov-Bohm property [24]. The kinetic effect was first time found in an experiment in normal metal by D.Yu. Sharvin and Yu.V. Sharvin [28], and in superconductor above $T_{c}$ by Shablo et al. [29], and theoretically described in a ring geometry by Ambegaokar and Eckern [30].

Bogachek and Gogadze [31] considered flux quantization in two-dimensional disk due to edge electron orbits («whispering gallery» trajectories [32]) inside the disk what was shortly confirmed in an experiment in thin bulk $\mathrm{Nb}$ filaments [33]. This was the first experimental demonstration of oscillations with single-electron flux quantum $h c / e$ compared with two-electron flux quantum $h c / 2 e$ characteristic of superconductors. Similar effects in hollow cylinders, quantum dots and antidots, as well as in specific magnetic materials have been discussed in papers [34-37].

Landauer and Buttiker [38] calculated resistance of the ring at a condition when the current exceeds its maximal value or an ac component is added to the external magnetic field.

Imry and Shiren [39] thoroughly discussed condition of persistent current observation regarding the effect of elastic and inelastic scattering to Aharonov-Bohm effect in solids. Their conclusion was that two-dimensional flat disk (quantum dot) whose lateral dimension is larger than the perimeter of electron orbit in perpendicular magnetic field, and the mean free path of electron exceeds the dot diameter, de Haas-van Alphen oscillation will show up superimposed on Aharonov-Bohm persistent current oscillation, at very strong magnetic field such the electron Fermi energy $\varepsilon_{F}$ is larger than the Landau levels spacing $\hbar e B_{z} / m c$.

Zagoskin et al. [40,41] considered quantum $h c / e-$ periodic oscillations of conductance in wide ballistic point contacts (e.g., see Ref. 42) superimposed on the conductance jumps of $e^{2} / \pi \hbar$ and $2 \mathrm{e}^{2} / \pi \hbar$ height.

Phase transitions induced by the Aharonov-Bohm field have been discussed by Azbel [43], Krive and Naftulin [44].

Bogachek, Krive, Kulik and Rozhavsky [45-48] considered realization of the Aharonov-Bohm effect in dielectric crystals in the state of charge density wave, in which the conductance by charged solitons dominates [49]. This was later discussed in a number of papers [50-53] and observed in an experiment [54].

Strong electron-electron and electron-ion correlations in metals are represented as the interplay of mutually interconnected phenomena such as Wigner crystallization [55], Coulomb blocade [56,57], and Luttinger liquid formation [58] which replaces the Fermi liquid of the non-interacting electrons. These phenomena have being discussed in the context of the Aharonov-Bohm effect. Glazman, Rudin and Shklowskii [59] considered quantum transport in a one-dimensional Wigner crystal; Maslov, Stone, Goldbart and Loss [60] studied Josephson currents in Luttinger liquid; Sundstrom and Krive [61] discussed the effect of Coulomb blockade on persistent current in the Luttinger-liquid ring; Moskalets $[62,63]$ discussed the effect of spin paramagnetism and of time-dependent magnetic field on Coulomb blockade in the ring; Krive, Sandstrom, Shekhter, Girvin and Jonson [64] thoroughly studied the persistent current and Aharonov-Bohm oscillations in the one-dimensional ring in a state of Wigner cryslal; Pletyukhov and Gritsev [65] investigated the persistent currents in Luttinger-liquid semiconducting rings; Krive, Palevsky, Shekhter and Jon- 
son discussed resonant tunneling and Coulomb blockade in quantum wires [66].

Persistent quantized currents in mesoscopic loops, cylinders, quantum dots and antidots as well as in regular and random ensembles of the dots have been discussed theoretically [67-69] and observed experimentally in many papers [70-78] etc. We refer especially to latest and most detailed contribution [79]. Much attention have been devoted to investigation of distinctions and similarities between the canonical and grand canonical averages in mesoscopic ensembles, and to the proportion in the orbital diamagnetic and paramagnetic magnitudes in corresponding susceptibilities [80-84].

Averin and Friedman [85,86] suggested using Aharonov-Bohm effect to study tunneling of quantum flux lines with the superconducting circuit incorporating Bloch transistor (the single-electron device [87]).

At the same period between 1970 and 1983, fundamental discovery of Quantum Hall Effect took place [88], and Laughlin [89] conjectured that the clean hollow cylinder in orthogonal to each other and to the axis of symmetry of cylinder magnetic and electric fields would be a starting point for the explanation of the physical origin of the sharp plateaus in the Hall voltages observed by Klitzing et al. [88]. Zanchi and Montambaux [90] discussed similarity or imitation of Aharonov-Bohm effect in CDW system to the integer QHE. Sivan and Imry [91] discussed simultaneous appearance of Aharonov-Bohm and de Haas-van Alphen oscillations in a same quantum dot.

Aharonov-Bohm effect in crossed magnetic fields [92-96], and in the electric field perpendicular to magnetic field [97-102] have been discussed in a number of papers. Particular interest is in the electromotive and magnetomotive forces accompanying persistent currents, and in the fast controllable transitions between the quantum states required for bit manipulations in quantum computers.

The fundamental problem of mesoscopic physics is in the work extraction from the quantum states [103]. This problem can be formulated as transitions between the quasistationary persistent-current states [100], using of the hypothized quantum force in mesoscopic superconducting rings in magnetic field [104], and as the charge transfer or flux pumping in the adiabatic modulation of persistent current by the ac signals [105-108], and as the theory of stochastic pumps and reversible ratchets [109] and molecular motors in the stochastic environment [110].

Time-dependent behavior is an important problem in physics and control of mesoscopic Aharonov-Bohm devices [101,111-116]. Three-site discrete quantum structure $[99,117]$ proved to be an interesting configuration which may allow performing basic operations required for realization of qubit in hypothetical quantum computer. That is the only one known electronic instrument allowing to perform full coherent quantum transformation $(1,0)$ to $(0,1)$ (a bit flip)in a finite time interval.
Spontaneous quantum flux state in a system of odd number of electrons with the Kramers degeneracy in the ground state have been analyzed in papers [100-102,118] and $[140,141]$. It was proved [100] that the state survives inelastic scattering within the isolated Aharonov-Bohm loop but is suspected of weak lifting of the degeneracy due to electromagnetic radiation in the environment [119].

It is interesting to note that another type of spontaneous current have also been discussed [120-122] in superconductive mesoscopic rings with Josephson contacts between the unconventional superconductors in which the angle between the orientation of $d$-vector in adjacent sides of contact (as well as a Josephson phase itself) is considered as a quantum variable.

Interplay between the normal-metal and the superconducting manifestations of the Aharonov-Bohm effect becomes an intriguing option of the quantum theory of solids development. As early as in 1975, Bogachek, Gogadze and Kulik [123], and later Wei and Goldbart [124] noticed change of critical temperature versus magnetic flux oscillations from $h c / e$ to $h c / 2 e$ - periodic, i.e. the doubling of the period of flux quantization in superconducting cylinders due to quantum effects in the normal state, and Zhang and Price [125] detected $h c / e$ - periodic oscillation of current to flux susceptibility in superconducting $\mathrm{Al}$ in narrow temperature interval above the zero-field critical temperature, in an exact agreement with the prediction of [123].

The interplay of attractive and repulsive Coulomb interactions results in $h c / 2 e$ periodic oscillations of the energy versus flux in strongly coupled electronic systems $[126,127]$ regardless on whether the system is superconducting or not. In a system of small number of electrons, superconducting behavior depends on whether the number of electrons is even or odd [128]. In the grand canonic ensemble, in which the number of electrons is not fixed, the two-fluid model shows different periodicities in the behavior of superfluid and normal-fluid (that of single-charged excitations) $[129,130]$. And in the last (but not the least important) case of unconventional superconductors [131134], both the normal-metal Aharonov-Bohm oscillations are displayed together with the $h c / 2 e$ superconductive oscillations.

\section{One-dimensional normal metal ballistic ring in crossed magnetic fields}

One-dimensional flat ring allows for exact solution for the quantum energy states, persistent currents and electromotive or magnetomotive forces (torques) acting on the ring in presence of the Aharonov-Bohm magnetic flux and arbitrary distribution of the external magnetic field around the ring. In this case, orbital magnetism is mixed with the paramagnetic magnetism of mobile electrons inside the ring.

The simplest configuration shown in Fig. 3 includes thin solenoid piercing the ring and vertical magnetic field 


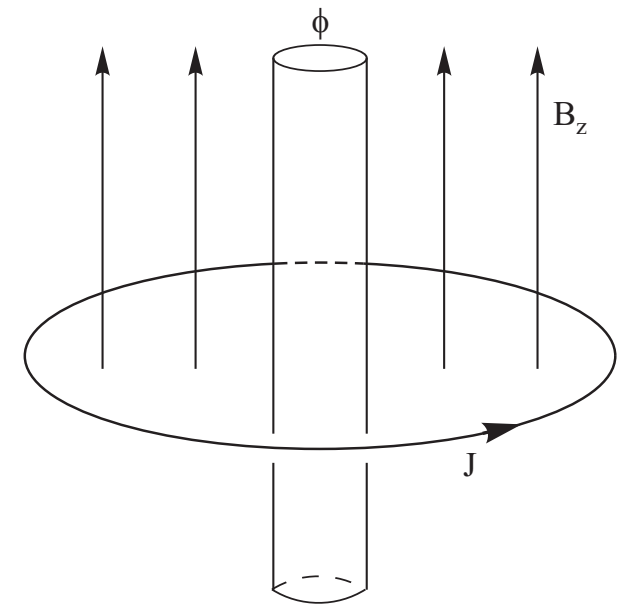

Fig. 3. Persistent current in presence of the Aharonov-Bohm flux $\phi$ and an external magnetic field $B_{z}$.

$B_{z}$ parallel to direction of solenoid. Hamiltonian of the system is

$$
H=\frac{\hbar^{2}}{2 m R^{2}}(\hat{n}-v)^{2} \sigma_{0}+\mu_{B} B_{z} \sigma_{3}
$$

where $R$ is radius of the ring and $\sigma_{i}$ the Pauli matrices:

$$
\sigma_{1}=\left(\begin{array}{ll}
0 & 1 \\
1 & 0
\end{array}\right), \sigma_{2}=\left(\begin{array}{cc}
0 & -i \\
i & 0
\end{array}\right), \sigma_{3}=\left(\begin{array}{cc}
1 & 0 \\
0 & -1
\end{array}\right), \sigma_{0}=\left(\begin{array}{ll}
1 & 0 \\
0 & 1
\end{array}\right)
$$

and $\hat{n}$ the operator $\hat{n}=\frac{1}{i} \frac{d}{d \vartheta}$, and $v=\phi / \phi_{0} \cdot \phi$ is the total magnetic flux threading the ring and $\phi_{0}=h c / e$ is the flux quantum of the normal metal. Solution of Eq. (11) for the energy eigenvalues gives

$$
\varepsilon_{n}=\frac{\hbar^{2}}{2 m R^{2}}(n-v)^{2} \pm \mu_{B} B_{z} .
$$

The diamagnetic current in the ring is received as a derivative $I=-c d E / d \phi$. Total magnetic moment of the ring including contribution from the current, $M_{1}=I S / c$, and paramagnetic contribution from spin in a magnetic field, $M_{2}=d E / d B_{z}$, is

$$
M_{z}=\frac{\mathrm{e} \hbar}{m c}\left(n-\frac{\phi}{\phi_{0}}\right)+\mu_{B} \sigma_{3} .
$$

Mention that $\phi$ is a total magnetic flux including contributions from the Aharonov-Bohm solenoid inside the ring and from the outside sources of magnetic field. $m$ is the effective mass, not necessary equal to the mass entering to the expression for the Bohr magneton $\mu_{B}$.

Another configuration is that when the outside magnetic field is perpendicular to Aharonov-Bohm field localized inside the ring (Fig. 4) what corresponds to Hamiltonian

$$
H=\frac{\hbar^{2}}{2 m R^{2}}(\hat{n}-v)^{2} \sigma_{0}+\mu_{B} B_{x} \sigma_{1}
$$

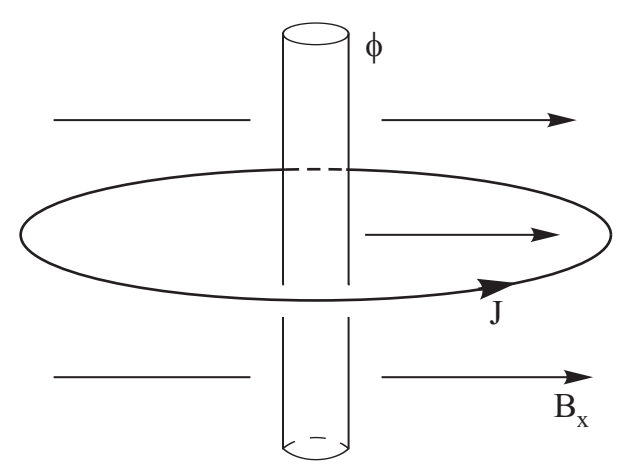

Fig. 4. Persistent current in configuration in which magnetic field is perpendicular to the direction of Aharonov-Bohm flux.

and to the equation for eigenenergies

$$
\varepsilon_{n}=\frac{\hbar^{2}}{2 m R^{2}}(n-v)^{2} \pm \mu_{B} B_{x}
$$

and for the magnetization

$$
M_{z}=\frac{e \hbar}{m c}\left(n-\frac{\phi}{\phi_{0}}\right), M_{x}= \pm \mu_{B} .
$$

Leaving for a while a discussion of physical implication of diamagnetic and paramagnetic contributions to the magnetization of the Aharonov-Bohm loop, we consider the cases of crossed fields with azimuthal (Fig. 5) and radial (Fig. 6) components of magnetic field orthogonal to the axes of symmetry of the ring. These configurations considered in the papers [92-95] within the Berry-phase technique $[135,136]$ valid in the adiabatic approximation, and as an exact solution [96] in static fields.

In case of azimuthal magnetic field $B_{\vartheta}$ at any point at the ring, Hamiltonian of the ring is

$$
H=\frac{\hbar^{2}}{2 m R^{2}}(\hat{n}-v)^{2} \sigma_{0}+\mu_{B} B_{\vartheta} \sigma_{\vartheta}
$$

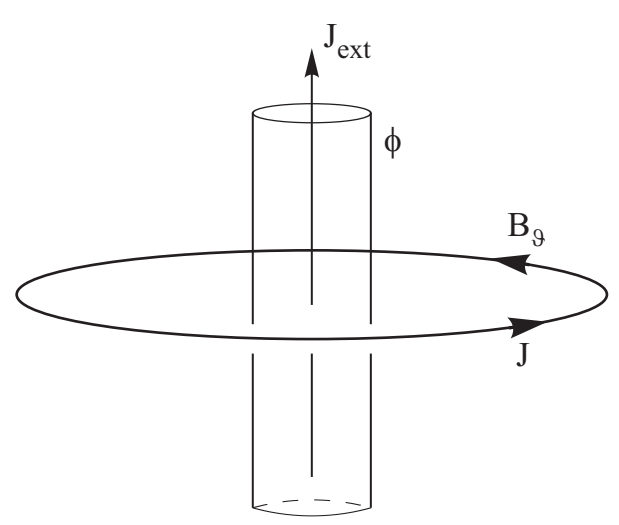

Fig. 5. Persistent current in a ring produced by the AharonovBohm flux $\phi$ and exsternal radial magnetic field $B_{\vartheta}$ emerging from the line current $I_{\text {ext }}$ in a wire along the symmetry axis of the ring $O Z$. 


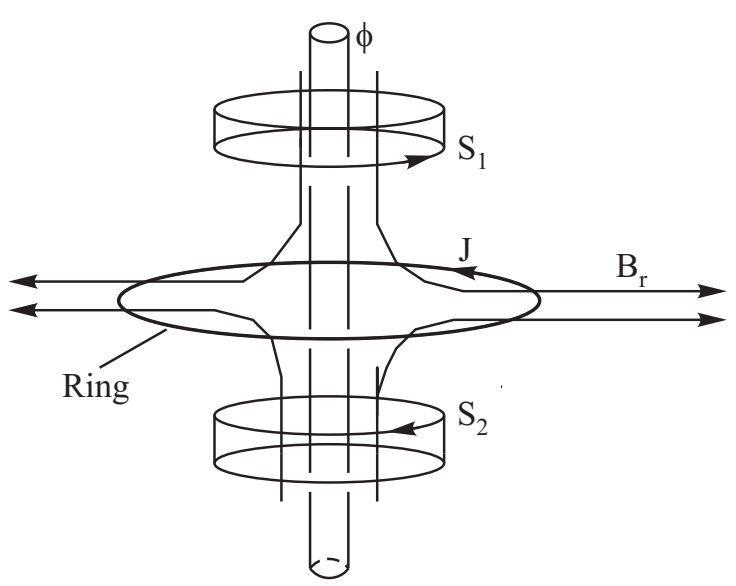

Fig. 6. Persistent current in a ring produced by the AharonovBohm flux $\phi$ in the inner cylinder and by the radial magnetic field $B_{r}$ created by a pair of magnetic coils $S_{1}$ and $S_{2}$ with opposite direction magnetization.

where $\sigma_{\vartheta}$ is a Pauli matrix:

$$
\sigma_{\vartheta}=\left(\begin{array}{cc}
0 & -i \mathrm{e}^{-i \vartheta} \\
i \mathrm{e}^{i \vartheta} & 0
\end{array}\right) .
$$

Psi-function $\Psi$ is a two-component vector

$$
\Psi=\sum_{n=-\infty}^{\infty}\left(\begin{array}{l}
\psi_{n} \\
\varphi_{n}
\end{array}\right) \exp (i n \vartheta)
$$

where for $\psi / \varphi$ bound components we have

$$
\begin{aligned}
& {\left[(n-v)^{2}-\varepsilon\right] \psi_{n}-i h_{\vartheta} \varphi_{n+1}=0,} \\
& i h_{\vartheta n}+\left[(n+1-v)^{2}-\varepsilon\right] \varphi_{n+1}=0,
\end{aligned}
$$

which gives the $n$th energy level

$$
\varepsilon_{n}=\left(n+\frac{1}{2}-v\right)^{2}+\frac{1}{4} \pm \sqrt{\left(n+\frac{1}{2}-v\right)^{2}+h_{\vartheta}^{2}}
$$

where energy is given in units of characteristic energy $\hbar^{2} / m R^{2}$, and $h_{\vartheta}$ is the value of magnetic field $B_{\vartheta}$ in these units.

The configuration of Fig. 6 is considered similarly to Eq. (18) in which last term replaces to $\mu_{B} B_{r} \sigma_{r}$ and Pauli matrix $\sigma_{r}$ is

$$
\sigma_{r}=\left(\begin{array}{cc}
0 & \mathrm{e}^{-i \vartheta} \\
\mathrm{e}^{i \vartheta} & 0
\end{array}\right)
$$

Respectively, Eq. (21) for energy will be

$$
\varepsilon_{n}=\left(n+\frac{1}{2}-v\right)^{2}+\frac{1}{4} \pm \sqrt{\left(n+\frac{1}{2}-v\right)^{2}+h_{r}^{2}} .
$$

Combining all paramagnetic interactions with external magnetic fields (Fig. 7), we receive the Hamiltonian

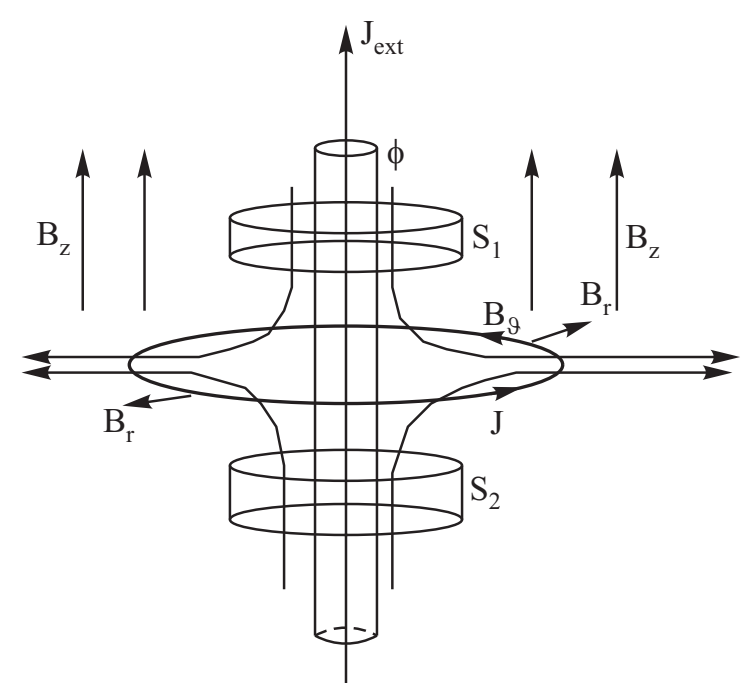

Fig. 7. Setup for observation of the Aharonov-Bohm effect in crossed magnetic fields $B_{z}, B_{\vartheta}$, and $B_{r}$ together with the magnetic flux $\phi$ in the inner solenoid.

$$
H=\frac{\hbar^{2}}{2 m R^{2}}\left(\hat{n}-\frac{\phi}{\phi_{0}}\right)^{2} \sigma_{0}+\mu_{B} B_{z} \sigma_{z}+\mu_{B} B_{\vartheta} \sigma_{\vartheta}+\mu_{B} B_{r} \sigma_{r}
$$

where $\phi$ is total magnetic flux from a thin solenoid inside the ring and from the $z$-component of external magnetic field penetrating through the full surface $4 \pi R^{2}$ enclosed by a ring; $B_{\vartheta}=2 I_{\text {ext }} / c R$ is the azimuthal component of the field at the ring, and $B_{r}$ the radial field component produced by two opposite-side solenoids inside the ring shown schematically in Fig. 7.

Energy levels of electron with the wave function

$$
\Psi=\left(\begin{array}{c}
\psi_{n} \\
\varphi_{n}
\end{array}\right) \mathrm{e}^{i n \vartheta} \mathrm{e}^{-i \varepsilon t}
$$

are

$$
\varepsilon_{n}=\left(n+\frac{1}{2}-v\right)^{2}+\frac{1}{4} \pm \sqrt{\left(n+\frac{1}{2}-v-h_{z}\right)^{2}+h_{\perp}^{2}}
$$

where $h_{\perp}=\sqrt{h_{\vartheta}^{2}+h_{r}^{2}}$ is a perpendicular field at the ring in units of $\hbar^{2} / 2 m R^{2}$.

\section{Conclusion and future perspectives}

Origin of persistent current in a normal-metal ring is relied on two postulates: (a) Lifting of the time-reversal symmetry in presence of vector potential $\mathbf{A}$, and (b) Rigidity of the wave function under change of the magnetic field. The rigidity, as it was for the first time introduced by London [2] in an explanation of the phenomenon of superconductivity of metals, means that $\Psi$ remained constant under change of magnetic field. The same is valid in case of persistent currents in normal metals since the wave function of an electron in the ring

$$
\Psi=\text { const } \exp (\text { in } \vartheta)
$$


is rigid (i.e., doesn't change) within certain interval of magnetic field

$$
\left(n-\frac{1}{2}\right) \phi_{0}<\phi<\left(n+\frac{1}{2}\right) \phi_{0}
$$

for a given $n$. In fact, we have here another type of superconductivity with a very small critical parameters, even smaller than in typical Josephson junctions (termed sometimes as a «weak superconductivity»).

The critical current in a $1 d$ ring is according to Eq. (14) and Eq. (1) is

$$
I_{A B}=e \hbar / 2 \pi m R^{2}
$$

compared to supercurrent in the same radius superconducting ring [137]

$$
I_{S C}=2 e \hbar N_{S} A / m R \sim \mathrm{e}^{3} / \hbar R,
$$

assuming superelectron concentration $N_{s} \sim N$ at $T=0$, and wire cross section $A$ of the order of the Bohr radius $a_{0}$ squared. Then the ratio of expression (30) to (31) is of order of $a_{0} / 2 \pi R$, the inverse of the number of electrons in the ring. The Aharonov-Bohm «superconductivity» is therefore the one-electron effect whereas in the BCS superconductivity all electronic pairs contribute to current collectively.

Aharonov-Bohm effect in crossed magnetic fields has an interesting property of anisotropy of magnetic susceptibility as discussed above in $\mathrm{Ch}$. 3 . The composition shown in Fig. 4 displays the magnetization component perpendicular to magnetic field. This means the presence of magnetomotive force (a torque)

$$
\mathbf{T}=\mathbf{M} \times \mathbf{B}
$$

acting on a ring. Such effect is well known in bulk monocrystalline normal metals where the weak rotation of the sample at change of static magnetic field serves as a most sensitive method [8] of studying the de Haas-van Alphen oscillations [6]. Similarly, levitating mesoscopic ring will display change of its orientation under control of the magnetic field.

The trend in studying quantum effects shifts in the last time to large molecular clusters rather than the artificial nanoscopic objects (quantum dots and antidots, rings, etc.). The observation of oscillating magnetization in large cyclic $\mathrm{Fe}_{10}$ clusters (Ferric Wheels) $[138,139]$ is one example of interesting possibilities existing in such structures. Flux quantization and persistent currents in Peierls insulators and charge-density-wave conductors with charge transfer mechanism by solitons and instantons is more developed theoretically $[45,46,50,53]$ but requiring further experimental efforts. Same concerns a study of Luttinger liquids in one dimension and their crystallization to onedimensional Wigner crystal $[64,65]$.
1. Diamagnetism and Paramagnetism, in: C. Kittel, Introduction to Solid State Physics, 7th edition, Ch.14. J. Willey, New York (1996).

2. F. London, Superfluids, Vol. 1. Dover Publ., New York (1961).

3. L.D. Landau, Zs. Phys. 64, 629 (1930).

4. E. Teller, Zs. Phys. 67, 311 (1931).

5. R.E. Peierls, Quantum Theory of Solids, Clarendon Press, Oxford (1955).

6. I.M. Lifshitz, M.Ya. Azbel, and M.I. Kaganov, Nauka Publ., Moscow (1971).

7. J.M. Ziman, Principles of the Theory of Solids, University Press, Cambridge (1964).

8. D. Shoenberg, Magnetic Oscillations in Metals, Cambridge University Press (1984).

9. J.H. van Leeuwen, J. Phys. (Paris) 2, 361 (1921).

10. K. Richter, D. Ullmo, and R.A. Jalabert, Phys. Rep. 276, 1 (1996).

11. E. Gurevich and S. Shapiro, J. Phys. I (France) 7, 807 (1997).

12. S.S. Nedorezov, Zh. Eksp. Teor. Fiz. 64, 624 (1973) [Sov. Phys. JETP 37, 317 (1973)].

13. I.O. Kulik, in: Quantum Mesoscopic Phenomena in Microelectronics, Ch. 7, p. 260, I.O. Kulik and R. Ellialtioglu (eds.), Kluwer Academic Publ., Dordrecht (2000).

14. B.I. Halperin, Phys. Rev. B25, 2185 (1982).

15. Y. Aharonov and D. Bohm, Phys. Rev. 115, 485 (1961); Phys. Rev. 123, 1511 (1961).

16. F. Hund, Calculation Concerning the Magnetic Behavior of Small Metallic Particles at Low Temperatures [reprinted from The Annalen Der Physik (Leipzig) 32, 102 (1938)]; Ann. Phys. (Leipzig) 5, 1 (1995).

17. H. Froelich, Proc. R. Soc. A223, 296 (1954).

18. N. Byers and C.N. Yang, Phys. Rev. Lett. 7, 2 (1961); Rev. Mod. Phys. 34, 694 (1962).

19. J.M. Blatt, Theory of Superconductivity, Academic Press, New York (1964).

20. M. Schick, Phys. Rev. 166, 404 (1968).

21. L. Gunther and Y. Imry, Solid State Commun. 7, 1391 (1969).

22. F. Bloch, Phys. Rev. B2, 109 (1970); Phys. Rev. Lett. 21, 1241 (1968); Phys. Rev. 166, 415 (1968); Phys. Rev. 137, A787 (1965).

23. R.B. Dingle, Proc. R. Soc. London, Ser. A, 212, 47 (1952); ibid. 216, 118 (1953); ibid. 219, 463 (1953).

24. I.O. Kulik, Pis'ma Zh. Eksp. Teor. Fiz. 11, 407 (1970) [JETP Lett. 11, 275 (1970)]; Zh. Eksp. Teor. Fiz. 58, 217 (1970) [Sov. Phys. JETP 31, 1172 (1970)].

25. M. Buttiker, Y. Imry, and R. Landauer, Phys. Lett. A96, 365 (1983)

26. B.L. Altshuler, A.G. Aronov, B.Z. Spivak, Pis'ma Zh. Eksp. Teor. Fiz. 33, 101 (1981) [JETP Lett. 33, 94 (1981)] [JETP Lett. 33, 94 (1981)].

27. A.G. Aronov and Yu.V. Sharvin, Rev. Mod. Phys. 59, 755 (1987).

28. D.Yu. Sharvin and Yu.V. Sharvin, Pis'ma Zh. Eksp. Teor. Fiz. 34, 285 (1981) [JETP Lett. 34, 272 (1981)].

29. A.A. Shablo, T.P. Narbut, S.A. Tyurin, and I.M. Dmitrenko, JETP Lett. 19, 246 (1974). 
30. V. Ambegaokar and U. Eckern, Phys. Rev. Lett. 65, 381 (1990).

31. E.N. Bogachek and G.A. Gogadze, Zh. Eksp. Teor. Fiz. 63, 1839 (1972) [Sov. Phys. JETP 36, 973 (1973)].

32. J.B. Keller and S.I. Rubinov, Ann. Phys. 9, 24 (1960).

33. N.B. Brandt, D.V. Gitsu, A.A. Nikolaeva, and Ya.G. Ponomarev, Pis'ma Zh. Eksp. Teor. Fiz. 24, 304 (1976) [JETF Lett. 24, 272 (1976)]; Zh. Eksp. Teor. Fiz. 72, 2332 (1977) [Sov. Phys. JETP 45, 1226 (1977)]; N.B. Brandt, E.N. Bogachek, D.V. Gitsu, G.A. Gogadze, I.O. Kulik, A.A. Nikolaeva, and Ya.G. Ponomarev, Fiz. Nizk. Temp. 8, 718 (1982) [Sov. J. Low Temp. Phys. 8, 358 (1982)].

34. E.N. Bogachek and G.A. Gogadze, Zh. Eksp. Teor. Fiz. Pis'ma Red. 17, 164 (1973).

35. E.N. Bogachek and U. Landman, Phys. Rev. B52, 14067 (1995).

36. O. Costa de Beauregard and J.M. Vigoureux, Phys. Rev. B9, 1626 (1974); O. Costa de Beauregard, Phys. Lett. A41, 299 (1972).

37. E.N. Bogachek and G.A. Gogadze, Zh. Eksp. Teor. Fiz. 67, 621 (1994).

38. R. Landauer and M. Buttiker, Phys. Rev. Lett. 54, 2049 (1985).

39. Y. Imry and N.S. Shiren, Phys. Rev. B33, 7992 (1986).

40. A.M. Zagoskin and I.O. Kulik, Fiz. Nizk. Temp. 16, 911 (1990) [Sov. J. Low Temp. Phys. 16, 533 (1990)].

41. E.N. Bogachek, A.M. Zagoskin, and I.O. Kulik, Fiz. Nizk. Temp. 16, 1404 (1990) [Sov. J. Low Temp. Phys. 16, 796 (1990)].

42. I.O. Kulik, Nonlinear Phenomena in Metallic Contacts, in: Quantum Mesoscopic Phenomena and Mesoscopic Devices in Microelectronics, Ch.1, p. 3, I.O. Kulik and R. Ellialtioglu (eds.), Kluwer Academic Publ., Dordrecht (2000).

43. M.Ya. Azbel, Phys. Rev. B48, 4592 (1993).

44. I.V. Krive and A. Naftulin, Nucl. Phys. B364, 541 (1991).

45. E.N. Bogachek, I.V. Krive, I.O. Kulik, and A.S. Rozhavsky, Phys. Rev. B42, 7614 (1990).

46. E.N. Bogachek, I.V. Krive, I.O. Kulik, and A.S. Rozhavsky, Zh. Eksp. Teor. Fiz. 97, 603 (1990).

47. A.S. Rozhavsky, Fiz. Nizk. Temp. 22, 462 (1996) [Low Temp. Phys. 22, 322 (1996)].

48. I.O. Kulik, A.S. Rozhavskii, and E.N. Bogachek, Pis'ma Zh. Eksp. Teor. Fiz. 47, 251 (1988).

49. W.P. Su, J.R. Schrieffer, and A.J. Heeger, Phys. Rev. Lett. 42, 1698 (1979).

50. G. Montambaux, Eur. Phys. J. B1, 377 (1998).

51. T. Matsuura, T. Tsuneta, K. Inagaki, and S. Tanda, Phys. Rev. B73, 165118 (2006).

52. Shi-Dong Liang, Yi-Hong Bai, and Bo Beng, Phys. Rev. B74, 113304 (2006).

53. I.V. Krive and A.S. Rozhavsky, Int. J. Mod. Phys. B6, 1255 (1992).

54. Yu.I. Latyshev, O. Laborde, P. Monceau, and S. Klaumunzer, Phys. Rev. Lett. 78, 919 (1997).

55. E.P. Wigner, Phys. Rev. 46, 1002 (1934).

1064
56. I.O. Kulik and R.I. Shekhter, Zh. Eksp. Teor. Fiz. 68, 623 (1975) [Sov. Phys. JETP 41, 308 (1975)].

57. D.V. Averin and K.K. Likharev, in: Mesoscopic Phenomena in Solids, B.L. Altshuler, P.A. Lee, and R.A. Webb (eds.), Elsevier, Amsterdam (1991).

58. S. Tomonaga, Progr. Theor. Phys. 5, 544 (1950); J.M. Luttinger, J. Math. Phys. 4, 1154 (1963).

59. L.I. Glazman, I.M. Rudin, and B.I. Shklovskii, Phys. Rev. B45, 8454 (1992).

60. D.L. Maslov, M. Stone, P.M. Goldbart, and D. Loss, Phys. Rev. B53, 1548 (1996).

61. P. Sandstrom and I.V. Krive, Ann. Phys. 257, 18 (1997).

62. M.V. Moskalets, Physica E4, 17 (1999).

63. M.V. Moskalets, Physica E8, 349 (2000).

64. I.V. Krive, P. Sandstrom, R.I. Shekhter, S.M. Girvin, and M. Jonson, Phys. Rev. B52, 23 (1995).

65. M. Pletyukhov and V. Gritsev, Phys. Rev. B70, 165316 (2004).

66. I.V. Krive, A. Palevski, R.I. Shekhter, and M. Jonson, Fiz. Nizk. Temp. 36, 155 (2010) [Low Temp. Phys. 36, 119 (2010)].

67. E.N. Bogachek, G.A. Gogadze, and I.O. Kulik, Fiz. Nizk. Temp. 2, 461 (1976) [Sov. J. Low Temp. Phys. 2, 228 (1976)].

68. E.N. Bogachek and I.O. Kulik, Fiz. Nizk. Temp. 9, 398 (1983) [Sov. J. Low Temp. Phys. 9, 202 (1983)].

69. E.N. Bogachek and U. Landman, Phys. Rev. B50, 2678 (1994).

70. V. Chandrasekhar, R.A. Webb, M.J. Brady, M.B. Ketchen, W.J. Gallagher, and A. Kleinsasser, Phys. Rev. Lett. 67, 3578 (1991).

71. E.M.Q. Jariwala, P. Mohanty, M.B. Ketchen, and R.A. Webb, Phys. Rev. Lett. 86, 1594 (2001).

72. L.P. Levy, Physica B169, 245 (1991).

73. D. Mally, C. Chapelier, and A. Benoit, Phys. Rev. Lett. 70, 2020 (1993).

74. S. Washburn, H. Schmid, D. Kern, and R.A. Webb, Phys. Rev. Lett. 59, 1791 (1987).

75. L.P. Levy, G. Golan, J. Dansmuir, and H. Bouchiat, Phys. Rev. Lett. 17, 2074 (1990).

76. S. Pedersen, A.E. Hansen, C.B. Sorensen, and P.E. Lindelof, Phys. Rev. B61, 5457 (2000).

77. K. Tsubaki, J. Appl. Phys, part 1, 3B, 1902 (2001).

78. N.O. Birge, Science 326, 244 (2009).

79. A.C. Bleszynski-Jayich, W.B. Shanks, B. Peaudecerf, E. Ginossar, F. von Oppen, and J.G.E. Harris, Science 326, 272 (2009) and arXiv.org/abs/0906.4780.

80. A.V. Balatsky and B.L. Altshuler, Phys. Rev. Lett. 70, 1678 (1993).

81. A. Kamenev and Y. Gefen, Phys. Rev. B49, 14474 (1999).

82. Y. Gefen, Y. Imry, and M.Ya. Azbel, Phys. Rev. Lett. 52, 129 (1984).

83. G. Montambaux, H. Bouchiat, D. Sigeti, and R. Friesner, Phys. Rev. B42, 7647 (1990).

84. B.L. Altshuler, Y. Gefen, and Y. Imry, Phys. Rev. Lett. 66, 88 (1991).

85. J.R. Friedman and D.V. Averin, Phys. Rev. Lett. 88, 050403 (2002).

Fizika Nizkikh Temperatur, 2010, v. 36, Nos. 10/11 
86. D.V. Averin, Fortshr. Phys. 48, 1055 (2000).

87. K. Likharev, in: Encyclopedia of Nanoscience and Nanotechnology 9, 865, H.S. Nalwa (ed.), American Sci. Publ. (2004).

88. K. von Klitzing, G. Dorda, and M. Pepper, Phys. Rev. Lett. 45, 494 (1980)

89. R.B. Laughlin, Phys. Rev. B23, 5632 (1981).

90. D. Zanchi and G. Montambaux, Phys. Rev. Lett. 77, 366 (1996).

91. U. Sivan and Y. Imry, Phys. Rev. Lett. 61, 1001 (1988).

92. D. Loss, P. Goldbart, and A.V. Balatsky, Phys. Rev. Lett. 65, 1655 (1990).

93. A. Stern, Phys. Rev. Lett. 68, 1022 (1992).

94. Y. Aharonov and A. Stern, Phys. Rev. Lett. 69, 3593 (1992).

95. D. Loss and P.M. Goldbart, Phys. Rev. B45, 13544 (1992).

96. I.O. Kulik, Physica B284, 1880 (2000).

97. I.O. Kulik, Persistent Current and Persistent Charge in Nanostructures, in Quantum Optics and the Spectroscopy of Solids, T. Hakioglu and A.S. Shumovsky (eds.), Kluver Acad. Publishers (1997).

98. I.O. Kulik, Physica B218, 252 (1996).

99. I.O. Kulik, T. Hakioglu, and A. Barone, Eur. Phys. J. B30, 219 (2002).

100. I.O. Kulik, Zh. Eksp. Teor. Fiz. 128, 1145 (2005) [J. Exp. Theor. Phys. 101, 999 (2005)].

101. I.O. Kulik, Phys. Rev. B76, 125313 (2007).

102. E. Zipper and M. Szopa, Acta Phys. Polonica A87, 79 (1995); Int. J. Mod. Phys. B9, 161 (1994).

103. A.E. Allahverdyan and T.M. Nieuwenhuizen, Phys. Rev. Lett. 85, 1799 (2000).

104. A.V. Nikulov, Phys. Rev. B64, 012505 (2001).

105. D.J. Thouless, Phys. Rev. B27, 6083 (1983).

106. F. Zhou, B. Spivak, and B. Altshuler, Phys. Rev. Lett. 82, 608 (1999).

107. B. Moskalets and M. Buttiker, Phys. Rev. B66, 035306 (2002).

108. M. Switkes, C.M. Marcus, K. Campman, and A.C. Gossard, Science 283, 1905 (1999).

109. N.A. Sinitsyn and I. Nemenman, Phys. Rev. Lett. 99, 220408 (2007).

110. V.Y. Chernyak and N.A. Sinitsyn, J. Chem. Phys. 131, 181101 (2009).

111. T. Swahn, E.N. Bogachek, Yu.M. Galperin, M. Jonson, and R.I. Shekhter, Phys. Rev. Lett. 73, 162 (1994).

112. I.E. Aronov, A. Grincwajg, M. Jonson, and R.I. Shekhter, Solid State Commun. 91, 75 (1994).

113. E.N. Bogachek, Yu.M. Galperin, M. Jonson, R.I. Shekhter, and T. Swahn, J. Phys. Cond. Matter 8, 2603 (1996).

114. I.V. Krive, I.A. Romavsky, E.N. Bogachek, and U. Landman, Phys. Rev. Lett. 92, 126802 (2004).
115. G.P. Berman, E.N. Bulgakov, D.K. Campbell, and I.V. Krive, Phys. Rev. B56, 10338 (1997).

116. Y. Makhlin and A.D. Mirlin, Phys. Rev. Lett. 87, 276803 (2001).

117. J. Mateo and J.M. Cervero, J. Phys. A 37, 2465 (2004).

118. M.Y. Choi, Phys. Rev. Lett. 71, 2987 (1993).

119. D. Loss and T. Martin, Phys. Rev. B47, 4619 (1993).

120. S. Yip, Phys. Rev. B52, 3087 (1995).

121. Yu.A. Kolesnichenko, A.N. Omelyanchouk, and S.N. Shevchenko, Fiz. Nizk, Temp. 30, 288 (2004) [Low Temp. Phys. 30, 213 (2004)].

122. Yu.A. Kolesnichenko, A.N. Omelyanchouk, and A.M. Zagoskin, Fiz. Nizk, Temp. 30, 714 (2004) [Low Temp. Phys. 30, 535 (2004)].

123. E.N. Bogachek, G.A. Gogadze, and I.O. Kulik, Phys. Status Solidi B67, 287 (1975).

124. T.-C. Wei and P.M. Goldbart, Phys. Rev. B77, 224512 (2008).

125. X. Zhang and J.C. Price, Phys. Rev. B55, 3128 (1997).

126. A. Ferretti, I.O. Kulik, and A. Lami, Phys. Rev. B47, 12235 (1993).

127. K. Czajka, M.M. Maska, M. Mierzjewski, and Z. Sledz, Phys. Rev. B72, 035320 (2005).

128. S.V. Sharov and A.D. Zaikin, Phys. Rev. B71, 014518 (2005).

129. J. Dajka, L. Machura, S. Rogozinski, and J. Luczka, Phys. Status Solidi B7, 2432 (2007).

130. J. Dajka, S. Rogozinski, L. Machura, and J. Luczka, Acta Phys. Pol. B38, 1737 (2007)

131. F. Loder, A.P. Kampf, T. Kopp, J. Mannhart, C.W. Schneider, and Y.S. Barash, Nature Phys., Adv. Online Publication 1, (2007).

132. F. Loder, A.P. Kampf, and T. Kopp, Phys. Rev. B78, 174 174526 (2008); F. Loder, A.P. Kampf, and T. Kopp, Phys. Rev. B78, 174526 (2008).

133. V. Juricic, I.F. Herbut, and Z. Tesanovic, Phys. Rev. Lett. 100, 187006 (2008).

134. V. Vakaryuk, Phys. Rev. Lett. 101, 167002 (2008).

135. Geometric Phases in Physics, in: Adv. Series in Physics, A. Shapere and F. Wilczek (eds.), World Scientific, Singapore (1989).

136. R. Onur Umucalilar, Berry's Phase (unpublished).

137. K.A. Matveev, A.I. Larkin, and L.I. Glazman, Phys. Rev. Lett. 89, 096802 (2002).

138. D. Gatteschi, A. Caneschi, L. Pardi, and R. Sessoli, Science 265, 1054 (1994).

139. K.L. Taft, C.D. Delfs, G.C. Papaefthymiou, S. Foner, D. Gatteschi, and J. Lippard, J. Amer. Chem. Soc. 116, 823 (1994).

140. S.V. Sharov and A.D. Zaikin, Physica E: Quantum Lowdim. Systems and Nanostructures 29, 360 (2005).

141. V.E. Kravtsov and M.R. Zirnbauer, Phys. Rev. B46, 4332 (1992). 